\title{
Transcatheter closure of ventricular septal defects using the Amplatzer Duct Occluder II device: a single-center experience
}

\author{
Tugcin Bora Polat, Esma Türkmen \\ Department of Pediatric Cardiology, Kemerburgaz University School of Medicine, Istanbul, Turkey
}

Adv Interv Cardiol 2016; 12, 4 (46): 340-347

DOI: 10.5114/aic.2016.63635

\begin{abstract}
A bstract
Introduction: Off-label use of different devices has been described for percutaneous closure of ventricular septal defects (VSD) because of the unacceptable rate of post-procedure heart block associated with special VSD devices.

Aim: To describe the early single-center clinical experience with closure of a VSD using the Amplatzer Duct Occluder II (ADO II) device in children.

Material and methods: Between May 2013 and June 2015, 26 patients between 13 days and 16 years of age underwent percutaneous closure of a VSD with an ADO II device at our institute. The decision to use the ADO II device was based primarily on lower risk of total atrioventricular block (TAVB) after VSD closure reported in the literature, reduction of the cost of the procedure and the need to close symptomatic VSDs in young patients.

Results: The location of the VSD was perimembranous in 21 patients, postsurgical residual in 2, midmuscular in 2 and apical muscular in 1. Complex procedures including newborn, combined procedures and postsurgical residual VSD were performed in 5 (19\%) patients. The median duration of follow-up was 12 months. The complete VSD closure rate was $81 \%$ immediately after the procedure, $85 \%$ at $24 \mathrm{~h}$, and $93 \%$ at the last follow-up. There was no device embolization. During the entire follow-up period, TAVB did not develop.

Conclusions: The ADO II constitutes a safe and effective therapeutic alternative for morphologically varied VSDs in all pediatric age groups. A larger range of sizes and configurations of this occluder may be required to successfully occlude a wider range of VSDs.
\end{abstract}

Key words: transcatheter closure, ventricular septal defect, Amplatzer Duct Occluder II.

\section{Introduction}

Amplatzer Duct Occluder (ADO) devices are patent ductus arteriosus (PDA) occlusion devices based on nitinol wire meshes. The ADO II device is a modification of the ADO I device as produced by AGA Medical Corporation. This device provides a solution for the closure of small to moderate-sized PDAs. It is shaped in sequential lobes characterized by two low-profile retention discs and a connecting waist. Flexibility of the articulations allows this device to simplify the treatment in a range of patients and specific defect anatomies that are more challenging [1-3].

Therefore, ADO II was also used in order to eliminate the risk of total atrioventricular block (TAVB) observed in follow-up using the perimembranous ventricular septal defect occluder (pmVSD-0) $[4,5]$. Theoretically, lower risk of TAVB is connected with a gentle construction of ADO II, namely thinner structure of the nitinol wire and lack of patches inside the occluder [6]. Ventricular septal defects larger than $6.5 \mathrm{~mm}$ and a distance less than $3 \mathrm{~mm}$ between the upper margin of the VSD and aortic valve are reported as two major limitations [7].

Nevertheless, there are not many articles in the literature describing the use of ADO II for VSD closure [7-9].

\footnotetext{
Aim

Here, we report our early follow-up results of percutaneous closure in all types of VSD including perimembranous, postsurgical residual, apical and midmuscular defects with an ADO II device in 26 subjects from newborn to adolescence.
} 


\section{Material and methods}

Between May 2013 and June 2015, we prospectively collected data on 26 children aged 13 days to 16 years of age with a VSD. The patients' general characteristics are reported in Table I. Patients were assessed by a standard echocardiographic protocol: all patients underwent transthoracic echocardiography (TTE) that was performed with a Vivid-3 echocardiography device
(GE Vingmed, Horten, Norway) using a transducer appropriate to each patient's size and body weight. The decision to use the ADO II device was based primarily on lower risk of TAVB after VSD closure reported in the literature [7-9], reduction of the cost of the procedure (the cost is $1 / 3$ that of regular ventricular septal occluders) and the need to close symptomatic VSDs in young patients.

Table I. Patient characteristics, echocardiographic features and follow-up

\begin{tabular}{|c|c|c|c|}
\hline Demographic data & Mean (SD) & Median (range) & $N(\%)$ \\
\hline Gestational age [years]: & $2.7(2.8)$ & $1.9(0.05-16)$ & \\
\hline Male & & & $17(65)$ \\
\hline Infant (< 2 years) & & & $16(62)$ \\
\hline Body weight [kg]: & $11.1(8.1)$ & $8.6(2.9-46)$ & \\
\hline Failure to thrive & & & $15(58)$ \\
\hline \multicolumn{4}{|l|}{ VSD type: } \\
\hline Perimembranous & & & $21(80)$ \\
\hline Muscular & & & $3(12)$ \\
\hline Postsurgical residual & & & $2(8)$ \\
\hline Complex procedures & & & $6(23)$ \\
\hline VSD size at right ventricle [mm] & $5(0.6)$ & $5.1(3.7-6.1)$ & \\
\hline Left atrium/aorta ratio & $1.9(0.4)$ & $2(1.3-2.6)$ & \\
\hline \multicolumn{4}{|l|}{ Procedural data: } \\
\hline VSD size at right ventricle side on TEE $[\mathrm{mm}]$ & $4.8(0.6)$ & $4.9(3.5-6.3)$ & \\
\hline Qp/Qs ratio & $2.3(0.4)$ & $2.4(1.6-3.3)$ & \\
\hline $\mathrm{Qp} / \mathrm{Qs}>2$ & & & $21(81)$ \\
\hline Pulmonary artery pressure [mm Hg] & $25(6.1)$ & $25(16-38)$ & \\
\hline Device waist diameter [mm] & $5.4(0.7)$ & $6(4-6)$ & \\
\hline Device length diameter [mm] & $4.3(1.1)$ & $4(2-6)$ & \\
\hline Device waist-VSD diameter difference [mm] & $0.6(0.4)$ & $0.9(0-1)$ & \\
\hline Fluoroscopic time [min] & $19(7)$ & $17(7-36)$ & \\
\hline Procedure time [min] & $73(24)$ & $67(25-113)$ & \\
\hline \multicolumn{4}{|l|}{ Complication data: } \\
\hline Major & & & $0(0)$ \\
\hline Minor & & & $7(26)$ \\
\hline \multicolumn{4}{|l|}{ Follow-up data: } \\
\hline Follow-up duration [months] & $11.6(6)$ & $12(2-26)$ & \\
\hline \multicolumn{4}{|l|}{ Procedural success rate: } \\
\hline Immediate & & & $21(81)$ \\
\hline At $24 \mathrm{~h}$ & & & $22(85)$ \\
\hline Overall & & & 24 (93) \\
\hline
\end{tabular}

VSD - ventricular septal defect. 


\section{Inclusion and exclusion criteria}

The criterion for inclusion in this study was clinical and/or echocardiographic evidence of a significant leftto-right shunt through a VSD. A left-to-right shunt was considered to be significant when at least 3 of the following were found: 1) cardiothoracic ratio on chest X-ray of $>0.55$; 2) left atrial enlargement, defined as a left atrial to aortic ratio $>1.5 ; 3$ ) left ventricular enlargement (left ventricular volume overload), defined as a left ventricular end-diastolic diameter $>2$ standard deviations (SD) above the mean for the patient's age; 4) overt heart failure, not improving with medications; 5) recurrent respiratory infections, defined as more than 6 events in the preceding 12 months [10]; 6) failure to thrive, defined according to Hamil et al. [11]; 7) mid-diastolic flow rumble at the apex on auscultation.

We excluded patients with infundibular VSDs, patients with an aortic valve prolapse, patients with VSDs larger than $6.5 \mathrm{~mm}$, and patients with other structural heart defects requiring surgery.

The protocol for the research project has been approved by a suitably constituted Ethics Committee of our institution within which the work was carried out, and it conforms to the provisions of the Declaration of Helsinki in 1995. Written informed consent was obtained from all parents or other surrogates for publication of this study and any accompanying images.

Percutaneous closure of a VSD was performed under general anesthesia with orotracheal intubation. The right femoral vein and left femoral artery were accessed with 5 -French sheaths. An intravenous heparin dose of $100 \mathrm{lU} /$ kg was applied after femoral artery access. The procedure was performed under fluoroscopic and transesophageal echocardiographic (TEE) control. Standard right and left cardiac catheterization, left ventriculography, and angiography of the ascending aorta were performed in all cases. The diameter of the VSD was measured on the left ventricular side and was calculated by integrating data from TEE and angiographic measurements. If possible, a device approximately $1 \mathrm{~mm}$ bigger than the smallest VSD diameter was chosen.

We preferred the antegrade approach via the right femoral vein in 24 patients and via the right internal jugular vein in two patients with posterior midmuscular VSDs. The VSD was crossed from the left ventricle using a 5-French right coronary artery catheter or a cut pigtail catheter with mounted 0.035 -inch angulated hydrophilic guidewire, and a 0.035 soft J-tipped Noodle guide wire (AGA Medical, Golden Valley, MN) was then advanced through the catheter into the pulmonary artery or the superior vena cava. The noodle guidewire was snared and exteriorized out through the femoral or right internal jugular vein to establish an arteriovenous loop. The long sheath was advanced from the femoral vein, through the VSD into the ascending aorta, and the device was advanced in the long sheath. A 5-French Pigtail catheter was placed retrogradely into the LV. Under TEE, fluoroscopy and LV cine angiographic guidance, the distal disc of the device was deployed just below the aortic valve and the whole system was pulled to the entrance of the defect at the left ventricular side, and finally the proximal disc was opened at the right ventricular side. New trivial aortic regurgitation was developed before releasing the device in a few cases with the upper margin of the VSD to the aortic valve distance $<3 \mathrm{~mm}$. In those cases, in order to avoid the device prolapse into the left ventricular outflow tract, the retentional disc at the left ventricle side was partially released and formed a "bubble shape". This compact form allowed the whole delivery system to withdraw to the interventricular septal (IVS) aneurysm. Thereafter, the proximal part of the device was opened. Finally, the correct position was confirmed and the device was released (Figure 1).

We closed an apical VSD in a newborn using the antegrade approach without establishing an arteriovenous loop. A 5-F multipurpose catheter and 0.035-inch angulated hydrophilic guidewire were used to cross the VSD, and a 0.014-inch guidewire was then advanced gently and smoothly into the aorta. The long sheath was advanced from the right ventricle, through the VSD into the left ventricular apex, and the device was advanced in the long sheath. The whole system was pulled under TEE guidance to the entrance of the interventricular septum, and the distal disc was opened. At the beginning, the distal disc had a "flattened shape" and failed to conform properly. By pulling repeatedly on the delivery cable with gentle pushing forward of the sheath against the disc, we finally succeeded in conforming the device in the mouth of the defect correctly. The proximal disc was then opened. Finally, the correct position was confirmed and the device was released (Figure 2).

\section{Statistical analysis}

Continuous variables were expressed as mean, median and standard deviation, while categorical variables were expressed as numbers and percentages. The objective of this article was to present the early experience with the Amplatzer Duct Occluder II for the treatment of VSD in a single-arm, single-center study; therefore, comparisons were not made. The SPSS 15.0 for Windows (SPSS, Chicago, USA) was used for the statistical computations.

\section{Results}

\section{Procedural data}

All subjects who met the inclusion criteria were sent to the catheterization laboratory with the intention to treat the defect percutaneously. Twenty-six patients (17 male) were eligible for the procedure (Table II). Their age ranged from 13 days to 16 years (me- 

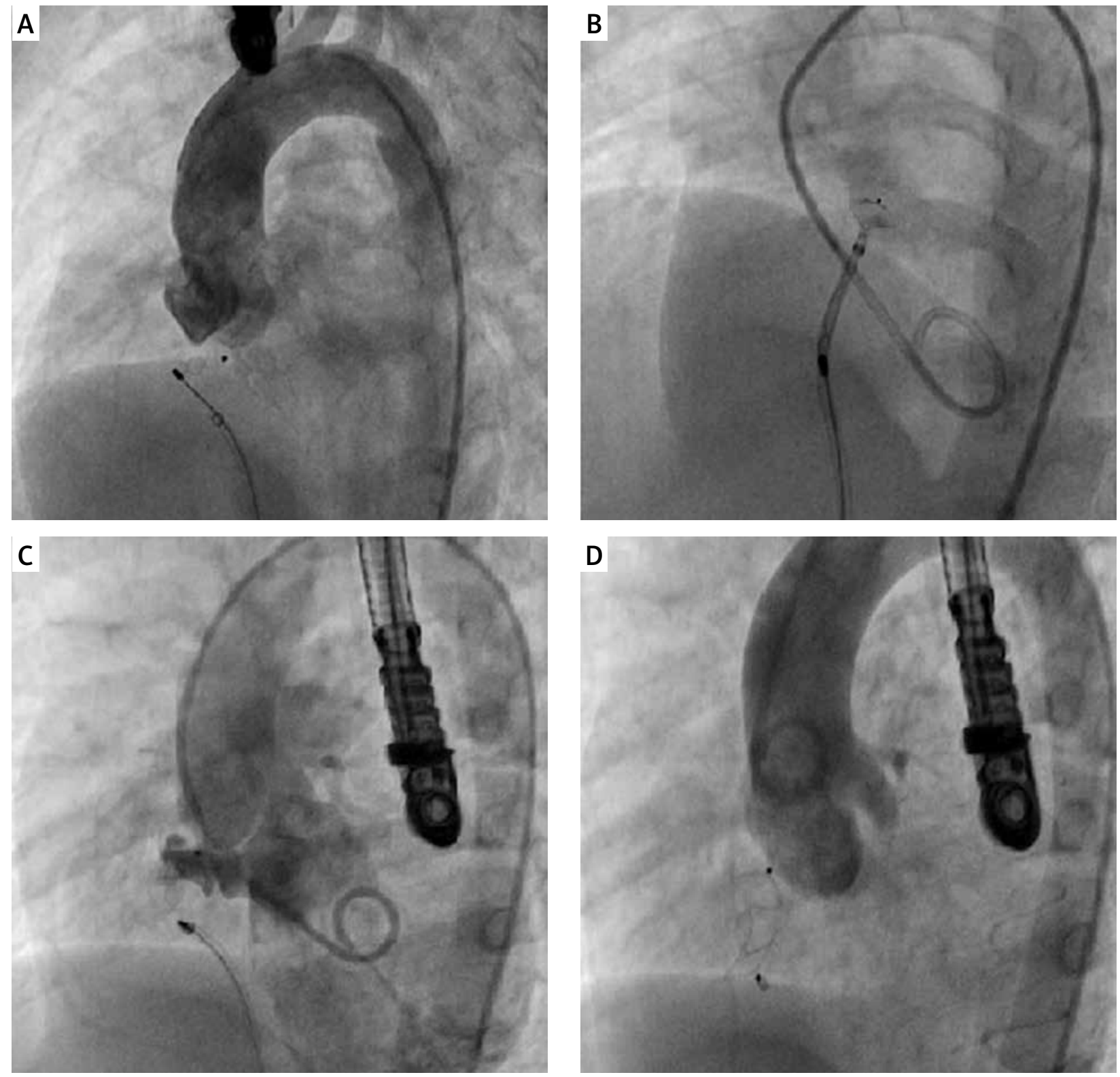

Figure 1. Strategy where upper margin of VSD to aortic valve distance $<3 \mathrm{~mm}$. A - Distal disc failed to conform properly, protruded into the left ventricular outflow tract, B - having half released the retentional ventricular disk, the distal disc formed a "bubble shape", C - this compact form allowed the whole delivery system to withdraw to the IVS aneurysm, $\mathbf{D}$ - the correct position was confirmed and the device released

dian: 1.9 years), and weight ranged from 2.9 to $46 \mathrm{~kg}$ (median: $8.6 \mathrm{~kg}$ ). The location of VSDs were perimembranous in 21 patients, postsurgical residual in 2, midmuscular in 2 and apical in 1 . Fourteen (53\%) of them had aneurysmal tissue around the VSD. The median VSD diameter on TEE was 4.9 (range: 3.5-6.3) $\mathrm{mm}$, the median device waist diameter was 6 (range: 4-6) $\mathrm{mm}$ and the median device length 4 (range: 2-6) $\mathrm{mm}$. Devices used (device diameter/length in $\mathrm{mm}$ ) were $4 / 2$ $(n=1), 4 / 4(n=2), 5 / 2(n=1), 5 / 4(n=7), 5 / 6(n=1)$, $6 / 4(n=8)$, and $6 / 6(n=6)$. Median fluoroscopic and procedure time was $17 \mathrm{~min}$ (range: $7-36$ ) and $67 \mathrm{~min}$ (range: 25-113), respectively. The complete VSD closure rate was $82 \%$ immediately after the procedure, $89 \%$ at $24 \mathrm{~h}$, and $93 \%$ at the last follow-up (Table I). There was no device embolization.

\section{Complex procedures}

Complex procedures (newborn, combined procedures, postsurgical residual VSD) were performed in 6 patients (23\%) (case \# 2, 9, 10, 14, 24, 25). Three patients underwent combined procedures including pulmonary valve dilatation in 1 and patent ductus arteriosus closure in 2. Two patients with postsurgical residual VSD and 

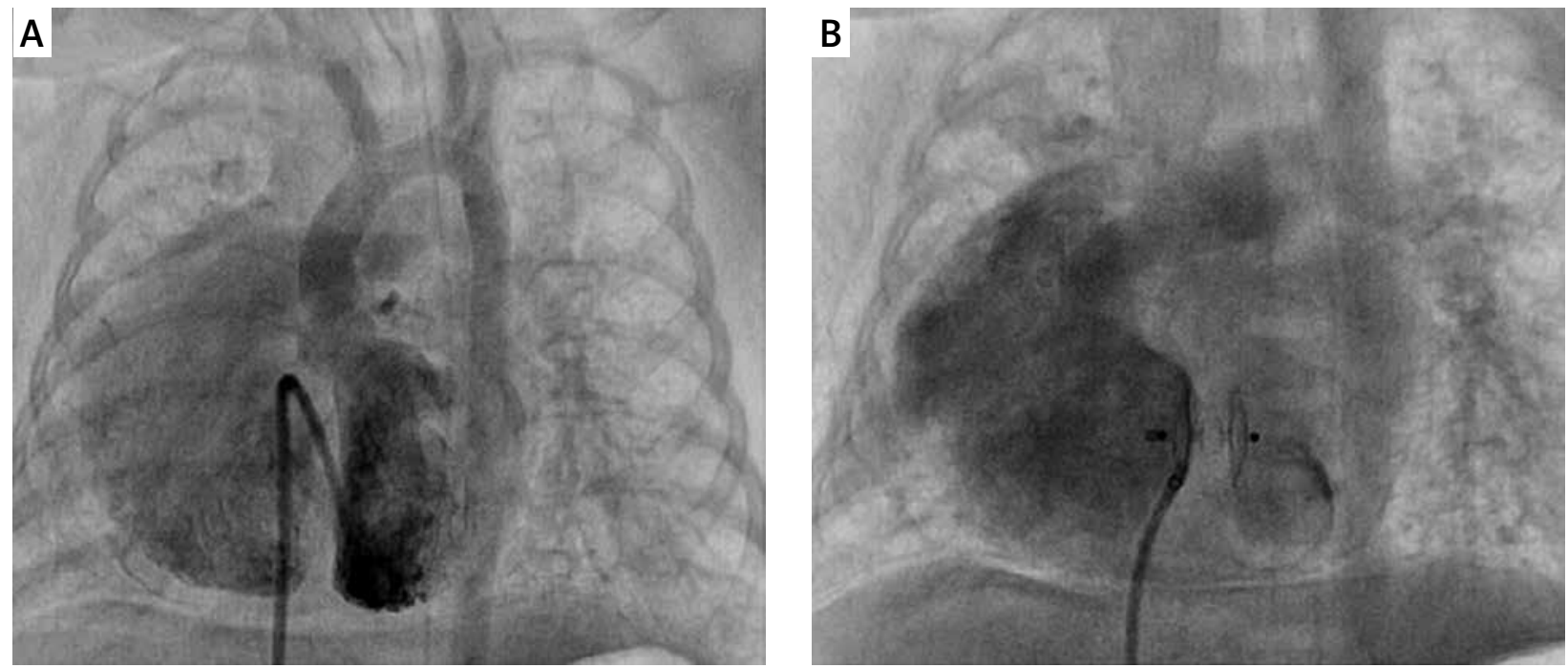

Figure 2. Apical large VSD caused extubation failure in a newborn with dextrocardia and situs solitus. A - 5-Fr multipurpose catheter was used to cross the $6 \mathrm{~mm}$ apical VSD, B - defect was closed with 6/4 mm ADO II device

1 newborn with dextrocardia, situs solitus and apical large muscular VSD underwent percutaneous closure.

\section{Complications}

There was no incidence of left bundle branch block, $\mathrm{P}-\mathrm{Q}$ prolongation, or TAVB during the follow-up evaluation. In 2 cases, the device dislodged to the right ventricle before release. Procedures were repeated all over in both, and, finally, the correct positions were confirmed and the devices were released. These 2 patients developed new trivial aortic regurgitation (AR) (case \# 6, 22). Five children had moderate tricuspid regurgitation (TR) immediately after the procedure. Three of them had pmVSD with inlet extension, and in all cases we preferred $6 \mathrm{~mm}$ device length diameter because of deep aneurysmal tissue covering the VSD. There was no incidence of femoral pulse loss.

\section{Follow-up}

Follow-up data were available for all patients. The median duration of follow-up was 12 months (range: 2-26 months). No deaths or cases of endocarditis occurred. Most children ( $n=15)$ were underweight before the procedure (less than the third centile for age and sex) and had complete recovery of growth (from $10^{\text {th }}$ percentile up to $50^{\text {th }}$ percentile during follow-up). Subjects with frequent respiratory infections had no significant recurrences. Left ventricular dimensions returned to normal in all subjects. A reduction of new existing TR by at least 1 grade was seen in all children the day after VSD closure and aortic regurgitation in 2 patients at the end of 1-month follow-up. Routine Holter ECG was performed at 6 months after the procedure in all patients and showed no signs of conduction disorder. During the entire follow-up period, TAVB did not develop.

\section{Discussion}

Some severe sequelae that may affect the outcome of the interventional approach to congenital heart disease are receiving increased attention from researchers $[12,13]$.

Total atrioventricular block is one of the most serious events and is reported to occur in $5-22 \%$ of subjects after perimembranous VSD closure using a PMVSD-O device $[4,14]$. It is difficult to ascertain the exact cause of heart block in such cases, but young age, low body weight, presence of a ventricular septal aneurysm, device oversizing and direct device compression trauma are the most probable risk factors $[15,16]$. Even TAVB was reported as a late event in some cases associated with a subsequent inflammatory reaction or scar formation in the conduction tissue $[4,14]$.

In the present study, we closed 21 perimembranous VSDs, 14 of which had aneurysmal tissue around the VSD. Our patients are mostly infant and underweight (Table I). Our subjects had the same risk factors besides the type of device. However, there was no incidence of TAVB immediately after the procedure or during the follow-up evaluation.

Unlike the PMVSD-O device, ADO II has a connecting waist of variable diameter and length for positioning within the VSD. Flexibility of the articulations and lack of patches inside the occluder allow this relatively soft device to adapt to the patient's anatomy rather than distorting the anatomy to its shape. Occluder design allows delivery with 4 or $5 \mathrm{Fr}$ delivery catheters with minimal local tissue and vascular injury [1-3].

The retention discs of ADO II are symmetrical and extend $3 \mathrm{~mm}$ radially around the connecting waist. Therefore, Koneti et al. [7] excluded all cases with the upper margin of the VSD to aortic valve distance less than 


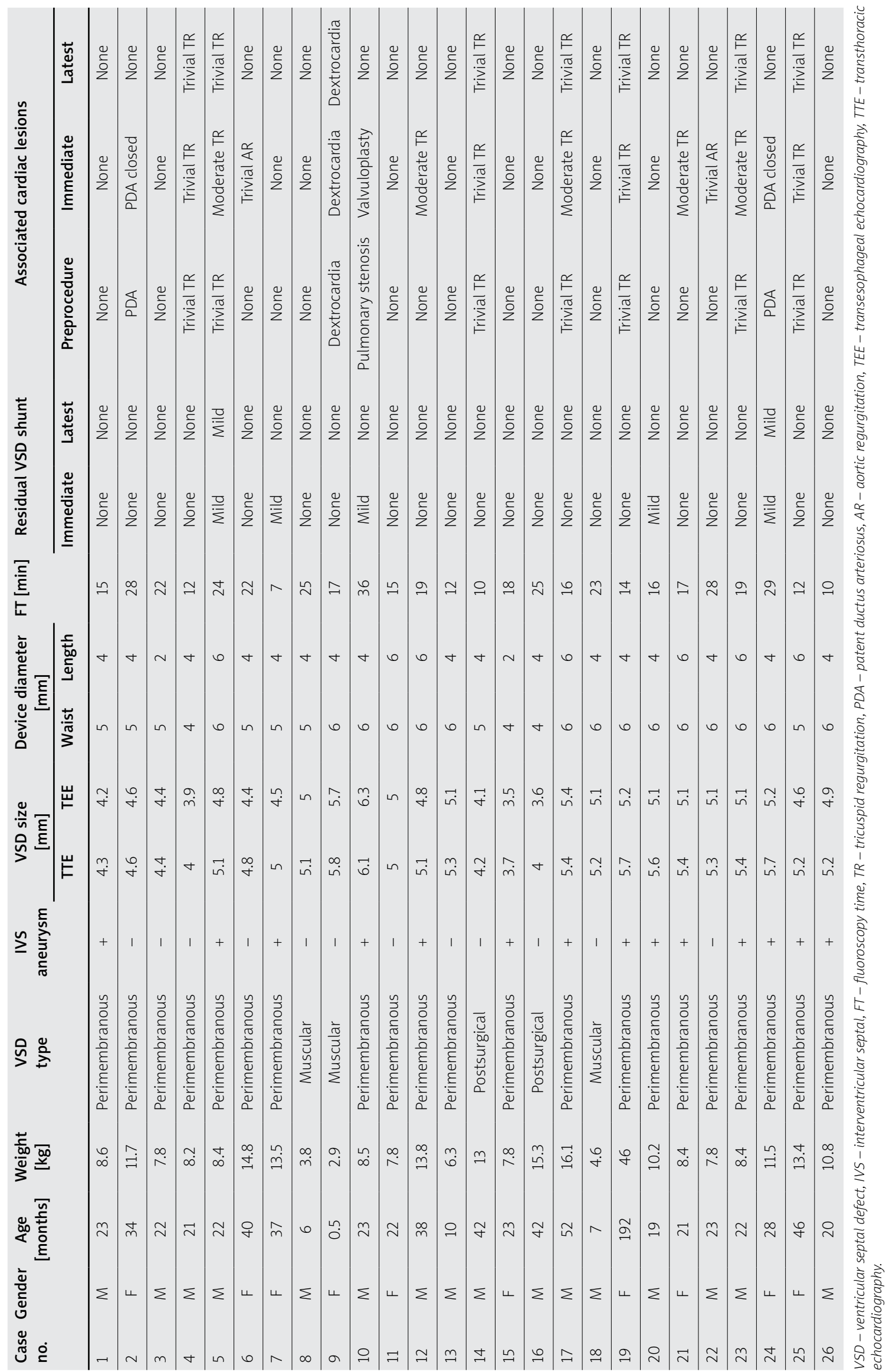


$3 \mathrm{~mm}$. At the beginning of our experience, we also used the ADO-II in 5 selected patients who had a distance of at least $3 \mathrm{~mm}$ between the superior rim of the defect and the aortic valve. However, our sixth case had a VSD with only 1 to $2 \mathrm{~mm}$ between the defect and the aortic valve. Thus, the device was pulled deep into the aneurysmal tissue covering the VSD before release in order to prevent protrusion of the distal disc to the aortic valve. This maneuver stretched the device, and finally the device dislodged to the right ventricle. The procedure was repeated all over again several times. At last, having half released the retentional ventricular disk, the proximal disc formed a "bubble shape". This compact form allowed the whole delivery system to withdraw to the IVS aneurysm. Since then, the indications for percutaneous closure have been expanded to include cases with less than $3 \mathrm{~mm}$ between the defect and the aortic valve, particularly cases having aneurysmal tissue (case \# 22).

Koneti et al. reported that the complete VSD closure rate was $78 \%$ immediately after the procedure as documented by left ventricular angiography and transthoracic echocardiography, $92 \%$ at $24 \mathrm{~h}$, and $94 \%$ at median follow-up duration of 14 months. Consistent with this, in our series of 26 patients the rate of successful implantation was $93 \%$ at median follow-up duration of 12 months.

Extubation failure necessitated VSD closure in a 13day-old term newborn (case \# 14). We firstly evaluated hybrid therapy to close a $5.7 \mathrm{~mm}$ apical VSD. However, it would be difficult to access the right ventricle with classic median sternotomy due to dextrocardia with situs solitus. Classic median sternotomy might allow us to access the left ventricle, and inserting a sheath into the left ventricle might cause injury to left ventricle structures. We easily crossed the large apical VSD using a 5-French multipurpose catheter and 0.035 -inch angulated hydrophilic guidewire antegradely, and closed the defect with a 6/4 mm ADO II device without complications. Twenty-four hours after the procedure, the newborn was extubated. To the best of our knowledge, this is the youngest patient to have undergone transcatheter device closure of a VSD with an ADO II device.

Five children had moderate TR immediate after the procedure. We felt that the newly released ADO II device, particularly the long ones, might interfere with the tricuspid valve leaflets and chordae. Nevertheless, all patients recovered at early follow-up. We concluded that retention discs with articulations allowed the relatively soft device to adapt to the patient's anatomy and would potentially interfere with the normal movement of the tricuspid valve soon after the procedure. Two children developed new trivial AR (case \# 6, 22). However, in both cases, the device dislodged in the right ventricle before release. Procedures were repeated all over several times in one and three times in the other. We considered that new exist- ing AR occurred due to minimal local tissue injury rather than direct device compression.

Although the techniques of percutaneous pmVSD closure appear to be safe in the short-term follow-up, it is not known whether they are safe in the very long term, whereas the TAVB was reported as a late event after percutaneous pmVSD closure in some studies [4, 10]. Finally, this is a prospective study with a small number of patients; a larger prospective study would be welcome to further assess the results obtained in our study. Consistent with this, we limited the follow-up results of all types of VSDs besides perimembranous ones.

\section{Conclusions}

ADO II is highly effective at providing occlusion of specific VSD types that are more challenging. Occluder design allows this device to simplify the treatment with excellent success and closure rates, no mortality, and very low morbidity. Availability of a larger range of sizes and configurations of this occluder might increase its applicability, allowing percutaneous closure of a wider range of VSDs.

\section{Conflict of interest}

The authors declare no conflict of interest.

\section{References}

1. Thanopoulos BV, Eleftherakis N, Tzannos K, et al. Further experience with catheter closure of patent ductus arteriosus using the new Amplatzer duct occluder in children. Am J Cardiol 2010; 105: 1005-9.

2. Baspinar O, Irdem A, Sivasli E, et al. Comparison of the efficacy of different-sized Amplatzer duct occluders (I, II, and II AS) in children weighing less than $10 \mathrm{~kg}$. Pediatr Cardiol 2013; 34: 88-94.

3. Forsey J, Kenny D, Morgan G, et al. Early clinical experience with the new Amplatzer Ductal Occluder II for closure of the persistent arterial duct. Catheter Cardiovasc Interv 2009; 74: 615-23.

4. Butera G, Carminati M, Chessa M, et al. Transcatheter closure of perimembranous ventricular septal defects. Early and long-term results. J Am Coll Cardiol 2007; 50: 1189-95.

5. Butera G, Carminati $M$, Chessa $M$, et al. Investigators of the European VSD Registry. Transcatheter closure of congenital ventricular septal defects: results of the European registry. Eur Heart J 2007; 28: 2361-8.

6. Wierzyk A, Szkutnik M, Fiszer R, et al. Transcatheter closure of ventricular septal defects with nitinol wire occluders of type patent ductus arteriosus. Postep Kardiol Interw 2014; 10: 21-5.

7. Koneti NR, Sreeram N, Penumatsa RR, et al. Transcatheter retrograde closure of perimembranous ventricular septal defects in children with the Amplatzer duct occluder II device. J Am Coll Cardiol 2012; 60: 2421-2.

8. Zhao PJ, Yu ZQ, Gao W, et al. Efficacy of the transcatheter closure of perimembranous and muscular ventricular septal defects with the Amplatzer duct occluder II. Zhonghua Xin Xue Guan Bing Za Zhi 2012; 40: 817-20. 
9. Kanaan M, Ewert P, Berger F, et al. Follow-up of patients with interventional closure of ventricular septal defects with Amplatzer Duct Occluder II. Pediatr Cardiol 2015; 36: 379-85.

10. Burgio GR. II bambino con infezioni ricorrenti. In: Immunologia Pediatrica. Plebani A (ed. ) Mc Graw Hill Libri Italia, Milano 1998; 17.

11. Hamil PV, Drizd TA, Johnson CL, et al. Physical growth: National Center for Health Statistics percentiles. Am J Clin Nutr 1979; 32: 607-29.

12. Noble S, Ibrahim R. Percutaneous interventions in adults with congenital heart disease: expanding indications and opportunities. Curr Cardiol Rep 2009; 11: 306-13.

13. Chessa M, Carrozza M, Butera G, et al. The impact of interventional cardiology for the management of adults with congenital heart defects. Catheter Cardiovasc Interv 2006; 67: 258-64.

14. Nehgme RA, Huddleston AR, Cheatham JP. Progression to late complete atrioventricular block following Amplatzer device closure of atrial septal defect in a child. Pediatr Cardiol 2009; 30: 367-70.

15. Walsh MA, Bialkowski J, Szkutnik M, et al. Atrioventricular block after transcatheter closure of perimembranous ventricular septal defects. Heart 2006; 92: 1295-7.

16. Yip WC, Zimmerman F, Hijazi ZM. Heart block and empirical therapy after transcatheter closure of perimembranous ventricular septal defect. Catheter Cardiovasc Interv 2005; 66: 436-41. 